\title{
Optimizing Growth and Neurocognitive Development While Minimalizing Metabolic Risk in Preterm Infants
}

\author{
Katie Pfister · Sara Ramel
}

Published online: 17 August 2014

(c) Springer Science + Business Media New York 2014

\begin{abstract}
Early aggressive nutrition is associated with improved neurodevelopmental outcomes, and is increasingly becoming the standard of care for preterm infants. Additionally, more rapid growth while in the NICU has been linked to lower risk of abnormal motor and cognitive development. However, as increasing reports of the metabolic consequences of rapid growth in other populations (i.e. appropriately grown and growth-restricted term infants) become available, we must consider the possibility that these negative long-term health outcomes may also affect preterm infants. This becomes especially concerning as studies revealing the prevalence of disproportionate growth and increased adiposity upon discharge from the NICU are published. This article will review the available literature on the impact of nutrition, illness and growth during several different epochs (i.e. first weeks of life, later inpatient weeks and post-discharge) on both neurodevelopmental and metabolic outcomes in preterm infants.
\end{abstract}

Keywords Preterm · VLBW · ELBW · Nutrition · Growth $\cdot$ Development $\cdot$ Neurodevelopment

\section{Introduction}

As increasing numbers of preterm infants survive into adulthood, the goals of both research and clinical care must

\footnotetext{
K. Pfister · S. Ramel $(\bowtie)$

Division of Neonatology/Department of Pediatrics, University of Minnesota, East Building- MB628, 2450 Riverside Avenue, Minneapolis, MN 55454, USA

e-mail: sramel@umn.edu

K. Pfister

e-mail: pfis0019@umn.edu
}

shift from solely decreasing mortality to reducing longterm morbidity among survivors. Studies revealing the beneficial effects of improved nutrition and growth on later neurodevelopmental outcomes of very-low-birth-weight (VLBW) preterm infants are abundant. Continued research into the amounts, types and duration of fortification remains vital to optimizing outcomes in this vulnerable population. However, as more information becomes available on the detrimental metabolic effects of rapid growth in term infants born after intrauterine growth restriction (IUGR), it becomes tempting to apply these same principles to preterm infants who undergo extrauterine growth restriction (EUGR) while in the NICU and following discharge home. While studies documenting the disproportionate growth (increased weight for length) and increased early adiposity in the preterm population are relatively consistent, those documenting the long-term metabolic consequences of this are more conflicted. As neonatologists strive to improve neurodevelopmental outcomes with aggressive nutrition and catch-up growth, are we worsening other long-term risks? In this article, we will review the current knowledge available on the influence of nutrition, illness and growth on later neurodevelopmental and metabolic outcomes of preterm infants.

\section{Neurodevelopmental Outcomes}

Many variables, including birth weight, gestational age, intraventricular haemorrhage (IVH) and periventricular leukomalacia (PVL), nutrition and growth and illness, affect the neurodevelopmental outcomes of preterm infants. Birth weight and gestational age are highly associated with outcomes; however, the neonatologist has no control over these variables. As IVH and PVL rates 
improve, focus is shifting to nutritional status, illness and growth-variables that are both known to affect neurodevelopment and are more easily manipulated by neonatologists—as targets to improve outcomes.

\section{Influence of Nutrition}

The relationship between early nutrition and neurocognitive development has long been known. In 1972, Lubchenco et al. studied outcomes of a group of preterm infants and commented that the "postnatal feeding routine was crucial in the poor outcome of the children reported...but the importance of these data will only become clear when children who have received adequate intakes are evaluated [1]". Since that time, many studies have evaluated the effect of nutrition alone on neurodevelopment; however, only recently has "early aggressive" nutrition become the norm. In the highly metabolic, developing preterm brain, providing sufficient nutrients is crucial for normal development.

The nutrition of preterm infants during several time periods has been studied for the effect on long-term development and can be broken down into early nutrition (in the first few weeks of life, usually composed of TPN), later inpatient nutrition (mostly enteral feeds), and post-discharge nutrition. Nutrition during the early period has the strongest evidence towards its effect on neurodevelopment. As summarized by Lapillone et al. [2], adequate energy [3, 4], protein [4] and lipid [5] intakes during the first weeks of extrauterine life are all independently associated with improved neurodevelopmental scores at 12-18 months [2].

Nutrition beyond the first weeks of life has less of a clear impact on neurodevelopment. Lucas et al. have published several studies evaluating developmental outcomes of preterm infants who received either term formula or preterm formula (with higher protein and energy) from birth until they reached $2000 \mathrm{~g}$ or discharge. At 18-month follow-up, those who had received preterm formula had better psychomotor development [6]. At 7.5-8 years, follow-up of a subgroup of infants showed that the preterm formula group had higher verbal IQ scores, which was statistically significant for boys [7]. Ergenekon et al. [8•] reviewed their NICU's $</=32$ week infants' protein intake after they reached full enteral feeds. Infants who received $4-5 \mathrm{~g} / \mathrm{kg} / \mathrm{d}$ of protein (through extra supplementation) versus $3.5 \mathrm{~g} / \mathrm{kg} / \mathrm{d}$ (via standard fortified breast milk or preterm formula) had higher mental and psychomotor scores at 18 months corrected age. However, a previous Cochrane review concluded that although protein supplementation of breast milk leads to increased growth parameters, there are insufficient data to evaluate long-term neurocognitive outcomes, and more research is needed [9].

Although we know nutrient-enriched (higher protein and energy) feeds after discharge improve growth (see section below), there is little evidence that this improves neurocognitive outcomes. Fifteen trials were included in a 2012 Cochrane review comparing nutrient-enriched formula versus standard term formula following hospital discharge, and it did not find any significant neurodevelopmental differences at 18 months [10•]. Further, a 2013 Cochrane review states that data are insufficient to recommend feeding preterm infants fortified breast milk after discharge (versus unfortified); one trial included neurocognitive follow-up and found no difference in outcomes [1 $1 \bullet$. Despite this, the ESPGHAN Committee on Nutrition recommends the use of post-discharge nutrition until 40-52 weeks corrected age in infants who are discharged at suboptimal weight for age [12].

In summary, optimizing nutrition early on, i.e. during the first weeks of life and during hospitalization, will decrease poor neurocognitive outcomes in preterm infants. There is little evidence that nutrient-enriched post-discharge diet, as currently constituted and prescribed, improves neurodevelopmental outcomes. Therefore, further research is needed to determine if this is due to continued inadequate fortification, an inappropriately balanced strategy of fortification (energy vs. protein) or that we have already missed the crucial developmental window for intervening by the time infants leave the hospital. Until there is such evidence, it will be most important to optimize nutrition prior to discharge and potentially avoid the need for catching up accrued nutritional deficits later.

\section{Influence of Illness}

Common neonatal morbidities that are associated with poor neurocognitive outcomes in preterm infants include bronchopulmonary dysplasia (BPD), retinopathy of prematurity (ROP), necrotizing enterocolitis (NEC), IVH and infection $[13,14,15 \cdot]$. While IVH may cause direct injury to the developing brain, other etiologies likely affect the brain via associated systemic responses. Mitha et al. [14] found that among a large group of VLBW preterm infants, those with isolated early or late onset sepsis had increased odds of cerebral palsy (CP), and those with both early and late onset sepsis had an even higher risk. Along with BPD, brain injury, and ROP, Schlapbach et al. [15•] also showed proven sepsis to be independently associated with a threefold risk of developing $\mathrm{CP}$ and a twofold risk of worsened neurodevelopmental outcome at 2 years.

Illness likely influences neurodevelopment in several ways. First, systemic inflammatory response syndrome leads to white matter injury in the vulnerable preterm brain $[16,17]$. Second, illness is an independent, non-nutritional factor that affects linear growth (an index of organ growth), and thus neurodevelopment [18•]. This may be mediated through growth hormone $(\mathrm{GH}) /$ insulin-like growth factor 1 (IGF-1) axis; IGF-1 synthesis is suppressed by 
pro-inflammatory cytokines common during illnesses like NEC and sepsis [19]. IGF-1 is not only important for linear growth but also affects neuronal growth and differentiation [20, 21]. Further, infants are likely to suffer from both poor nutrition and high catabolic states during illness, which, as described above, influences neurocognitive development.

\section{Influence of Growth}

There is an abundance of new literature regarding neonatal growth patterns and later neurocognitive outcomes. Within these studies, growth is evaluated in many ways-weight, length, head circumference (HC), body mass index (BMI) and body composition. Across all variables, preterm infants often differ from their term counterparts at discharge. EUGR is common, as nearly half of preterm infants drop $>1 \mathrm{SD}$ in weight for age during their NICU admission [22].

Postnatal growth from birth to term in preterm infants is associated with neurodevelopmental outcomes. In 2006, Ehrenkranz et al. [23] demonstrated that increased postnatal weight and head growth velocity (during hospitalization) are associated with decreased risk of poor motor and cognitive neurodevelopmental scores and lower rate of $\mathrm{CP}$ at 2 years. Weight and head growth from birth to term also have a positive effect on cognitive and motor outcomes at 5 years [24].

Belfort et al. studied 613 infants born $<33$ weeks gestation to identify sensitive periods of postnatal growth relative to neurodevelopment. They found that birth to term weight, HC and BMI gains were associated with higher mental and psychomotor scores at 18 months corrected age; this was strongest for infants $<1250 \mathrm{~g}$ at birth. Linear growth during this time was not associated with developmental scores. However, from term to 4 months, weight gain and linear growth were associated with higher psychomotor scores. There were no measurements from 4-12 months corrected age associated with neurodevelopment at 18 months [25•*]. Our group evaluated infants born $<30$ weeks and found linear growth at 4 and 12 months corrected age to positively correlate with cognitive scores at 24 months but not psychomotor scores [18•]. Another study by Belfort et al. [26] followed 945 preterm participants born in the $1980 \mathrm{~s}$ (mean GA at birth 33 weeks) in the Infant Health and Development Program (IHDP) through 18 years and found more rapid linear growth from term to 4 months corrected age to be positively associated with full-scale IQ at 8 and 18 years. BMI gain during this time period did not benefit cognition. While all three of these studies suggest linear growth after term benefits longterm development, they differ in whether that benefit is cognitive or motor and the exact timing of the measurements. This may be related in part to differences in the study populations, as each study looked at slightly different GAs (mean birth GA of 26.8 weeks [18•] vs. 30 weeks [25••] vs.
33 weeks [26]). As brain development continues throughout gestation and beyond, the timing of nutritional deprivation and illness, as well as the duration, lead to different areas of the brain being influenced more or less by stressful events, and therefore lead to different clinical manifestations. Further, one of the studies looked at school age outcomes, while the others were at 18-24 months. Future studies of comparable populations and well-defined time periods, as well as frequent measures will help clarify these differences.

While BMI has been used to estimate body composition of preterm infants, true composition using air displacement plethysmography is now also available. Preterm infants have higher adiposity and lower fat-free mass (FFM) at term compared to their term-born counterparts $[18 \bullet, 27$, 28]. Our group studied a cohort of 16 preterm (mean GA 30.4 weeks) infants and found increased FFM at discharge, and 4 months corrected age were associated with faster speed of visual processing at 4 months corrected age [29]. Further studies of body composition and neurocognitive development are needed.

In summary, current research suggests that birth to 4 months corrected age is the most sensitive time period for growth and its relation to neurocognitive outcomes in AGA preterm infants. From birth to term, gains in weight, length, HC, BMI and FFM are associated with improved outcomes. While after term, linear growth most strongly correlates with improved cognitive outcome. Catch-up growth in weight after 4 months appears to have minimal impact on long-term developmental outcomes; however, further studies are needed to investigate the influence of FFM, fat mass (FM) and linear gains during this time period.

\section{Metabolic Outcomes}

Obesity and metabolic syndrome are increasingly significant problems around the world, and rates of obesity have more than doubled since the 1980's [30]. Adult lifestyle choices contribute largely to these statistics; however, significant research is now focused on the influence of early life events on these later outcomes. Early reports in the 1990's by Barker et al. noted that infants who were born small had increased incidence of cardiovascular disease as adults [31]. These initial data were obtained from infants who were born at term and had undergone IUGR. As preterm infants are also born small, and often undergo growth restriction after birth (EUGR), more recent literature has also looked at the influence of prematurity on later metabolic outcomes. Multiple large studies and systematic reviews have recently been published stating that preterm birth alone is associated with reduced insulin sensitivity [32], elevated blood pressure and resting heart rate [33, 34, $\left.35{ }^{\circ}\right]$ and slightly increased low-density lipoprotein [35•]. In 
this section, we will review the literature on the influence of growth, nutrition and illness on long-term obesity and metabolic outcomes in preterm infants.

\section{Influence of Nutrition}

While the positive influence of early nutrition on long-term neurodevelopmental outcomes is well documented, evidence linking nutritional provision to later metabolic outcomes is much more sparse and conflicted. There are an increasing number of studies looking specifically at short-term body composition changes with more aggressive nutrition. These studies have largely found that improved nutrition during the NICU stay and throughout early infancy lead to increased FFM gains without increased FM [36, 37]. We reported improved FFM gains, without increased FM gains, through 4 months to be associated with increased caloric intake throughout hospitalization [36]. Costa Orvay et al. [37] also found that aggressive nutritional supplementation once on full feeds ( $\sim 4.5 \mathrm{~g}$ protein and $\sim 150 \mathrm{kcals}$ ) led to increased FFM gains without increased FM gains. Roggero et al. recently reported improved HC and lower FM in preterm infants receiving fortified feeds (vs. term formula) for up to 6 months post-discharge as well [38•]. Studies looking at long-term outcomes are harder to come by and more conflicted. Ludwig-Auser found that preterm infants who received more aggressive nutrition ( $>70 \mathrm{kcals} / \mathrm{kg} /$ day and $>2.5 \mathrm{~g} / \mathrm{kg} /$ day of protein) during the first 2 weeks of life remained taller and heavier as adolescents, but did not have increased percent body fat or increased systolic or diastolic BP [39]. However, Singhal et al. [40] found fasting split proinsulin levels that are greater in those preterm infants receiving a nutrient-enriched diet until term. Interestingly, the levels in the preterm infants receiving fortified feeds were similar to the term control group, suggesting that fortification is not increasing the risk from baseline but rather returning it to baseline.

In summary, there is to date little to no evidence that early aggressive nutrition increases the risk of later adverse metabolic outcomes in AGA preterm infants. Increased provision of protein and calories early and continuing throughout early infancy improves FFM gains and, therefore, may actually reduce the long-term risk of metabolic disturbances later in life. Further study is needed into the type and duration of various types of parenteral and enteral supplementation needed to optimize outcomes.

\section{Influence of Illness}

Until recently the negative impact of illness on growth in preterm infants was attributed primarily to limited nutritional provision. However, the impact of inflammation on disproportionate growth is just beginning to be recognized. We have found that degree of illness during hospitalization negatively influences linear growth (a surrogate for FFM) out to 24 months of age corrected for prematurity [18•]. Additionally, we have found that infants with an increased severity of illness on their first day of life had less FFM at 4 months of corrected age [36]. Uthaya et al. [41] reported that at term corrected age, preterm infants have significantly decreased amounts of subcutaneous fat and significantly increased intraabdominal adiposity. The primary determinant of this increased abdominal adipose tissue was illness severity.

Given that decreased amounts of FFM, as well as increased amounts of abdominal adiposity, can be associated with decreased insulin sensitivity, it seems reasonable to hypothesize that early illness may play an important role in later risk of metabolic disease. Further study looking specifically at markers of inflammation and illness and controlling for nutritional intake will be important to further examine this issue.

\section{Influence of Growth}

Body composition of preterm infants when measured at term corrected age is characterized by diminished FFM and increased adiposity [27, 36, 42] compared to their term counterparts. The duration of this altered growth and the long-term implications remain unclear; however, recent data suggest that the differences may be short term. We have previously reported that differences in body composition between term and preterm infants have diminished by 4 months corrected age for prematurity [36]. Griffin and Cooke described preterm infants having significantly lower percent body fat by 3 months corrected age, and throughout the first year [43]. Decreased FM in preterm infants when compared to terms has also been reported at school age (4.8-6.6 years) [44] and at 8-12 years of age [45]. A recent meta-analysis additionally found no difference in percent fat mass between term and preterm infants throughout adulthood [35•]. Therefore, it appears that although preterm infants have significantly increased FM and lower FFM at discharge, these differences resolve, and in fact may reverse, as early as late infancy and remain this way through adulthood.

While it appears that differences in body composition are short-lived, the long-term metabolic ramifications of these early alterations are just beginning to be recognized. BMI gain from term to 18 months of age in preterm infants has been associated with increased odds of obesity at age 8 and 18 [26]. Casey et al. [46] also showed that high weight gain in the first year of life increases the risk of obesity at 8 years of age in preterm infants. Finally, Euser et al. [47] reported a positive association between weight gain from birth to 3 months post-term and from 3 months post-term to 1-year post-term and BMI at age 19 .

A number of studies (summarized by Lapillionne et al.) of preterm infants looking at early growth do not show 
associations between weight gain and later hypertension, glucose tolerance, insulin sensitivity or lipid profiles [2]. Rather, as in the term population, childhood weight gain and obesity are significantly associated with metabolic outcomes [2]. Irving et al. [48] evaluated a group of low birth weight infants born in the mid 1970's and found no association between weight gain throughout the first year of life and adult blood pressure. Two other studies of preterm infants ( $<32$ weeks) found that infant weight $\mathrm{z}$-scores were unrelated to blood pressure measurements in late adolescence $[49,50]$. Belfort et al. [51] found that weight gain from term to 1 year (only in infants $>32$ weeks gestation at birth) was associated with slightly increased BP $(1.1 \mathrm{~mm} \mathrm{Hg}$ higher systolic blood pressure) in childhood.

Additionally, Irving et al. [48] found no association between early weight gain and fasting glucose or lipid levels in adulthood. Another study by Fewtrell et al. [52] confirmed that childhood weight gain, rather than weight gain prior to 18 months of age, predicted insulin sensitivity in adolescence. Several studies have examined the influence of weight gain during infancy on later lipid values, and none have confirmed an association [53, 54].

There have been very few studies documenting the impact of early in-hospital growth (up to 40 weeks) in the preterm population on later obesity and metabolic outcomes. This information is especially important for several reasons, including that this is the time period where the neonatologist has the most control over nutritional intakes and also the time period that has been shown to be the most critical for improving neurodevelopmental outcomes. Therefore, optimizing early growth to avoid the need for significant catchup growth later in life seems the most beneficial strategy to both neurodevelopmental and metabolic outcomes. Additionally, growth must not be defined simply as weight gain, but rather linear growth and proportionality, and potentially body composition must be followed closely both during the hospitalization and beyond.

\section{Conclusions}

The relationships among nutrition, growth, illness, neurodevelopment and metabolic outcomes are especially complex among preterm infants. As we strive to improve longterm outcomes, it is important to consider later health (specifically cardiovascular and metabolic) as well as development. However, based on the above summary, it appears that we should not be simply applying the extensive literature on term IUGR infants to the care of preterm infants undergoing EUGR. Early aggressive nutrition improves neurodevelopment in preterm infants while minimally affecting metabolic outcomes. Increased provision of protein and calories early and continuing throughout early infancy improves FFM gains and, therefore, may actually reduce the long-term risk of metabolic disturbances later in life. Further, gains in all parameters from birth to term are associated with better neurodevelopment, with no evidence of negative impact on metabolic outcomes. Thus, it appears prudent that we continue to provide enhanced nutrition and optimize growth of preterm infants during hospitalization. After term, linear growth and BMI gains are associated with increased risk of obesity, with only linear growth providing cognitive benefit. Additionally, excess and disproportionate growth beyond early infancy ( $\sim 4$ months corrected age) increases risks of obesity and metabolic derangements with minimal to no neurodevelopmental benefit. Closely monitoring proportionality and potentially body composition appears even more important during these later time periods, and adjustment of nutrition accordingly will likely be beneficial.

Illness negatively affects neurodevelopmental and metabolic outcomes. Systemic inflammatory response leads to direct brain injury, while also affecting growth and development of the brain via the GH/IGF-1 axis. Illness leads to decreased FFM and linear growth as well as increased central adiposity, and decreased insulin sensitivity, all of which play a later role in metabolic disease. Unfortunately, as of yet, these are factors largely out of the neonatologist's control. Further research aimed at potentially enhancing the GH/IGF-1 system or decreasing inflammation is important as it may improve both long-term health and development.

Given the current literature, neonatologists should continue to optimize growth and nutrition early to avoid the need for later excessive catch-up growth, which is more likely to be detrimental to later metabolic outcomes. Further research into types and duration of nutritional supplementation, as well as methods of decreasing the influence of illness, will be essential to optimizing both long-term health and development.

Disclosure Katie Pfister and Sara Ramel declare that they have no conflict of interest.

Human and Animal Rights and Informed Consent This article does not contain any studies with human or animal subjects performed by any of the authors.

\section{References}

Papers of particular interest, published recently, have been highlighted as:

- Of importance

-• Of major importance

1. Lubchenco LO, Delivoria-Papadopoulos M, Butterfield LJ, et al. Long-term follow-up studies of prematurely born infants, I: relationship of handicaps to nursery routines. J Pediatr. 1972;80:501-8. 
2. Lapillonne A, Griffin IJ. Feeding preterm infants today for later metabolic and cardiovascular outcomes. J Pediatr. 2013;162(3 Suppl 1):S7-16.

3. Brandt I, Stickler EJ, Lentze MJ. Catch-up growth of head circumference of very low birth weight, small for gestational age preterm infants and mental development to adulthood. J Pediatr. 2003;142:462-8.

4. Stephens BE, Walden RV, Gargus RA, et al. First-week protein and energy intakes are associated with 18-month developmental outcomes in extremely low birth weight infants. Pediatrics. 2009;123:1337-43.

5. dit Trolli SE, Kermorvant-Duchemin E, Huon C, Bremond-Gignac D, Lapillone A. Early lipid supply and neurological development at one year in very low birth weight (VLBW) preterm infants. Early Hum Dev. 2012;88(Suppl1):S25-9.

6. Lucas A, Morley R, Cole TJ, et al. Early diet in preterm babies and developmental status at 18 months. Lancet. 1990;335:1477-81.

7. Lucas A, Morley R, Cole TJ. Randomized trial of early diet in preterm babies and later intelligence quotient. $\mathrm{Br}$ Med $\mathrm{J}$. 1998;317:1481-7.

8. • Ergenekon E, Soysal S, Hirfanoglu I, et al. Short- and long-term effects of individualized enteral protein supplementation in preterm newborns. Turk J Pediatr. 2013;55(4):365-70. Preterm formulas or human milk fortifiers may not have the ideal protein concentration for preterm infants. This study suggests that adding extra protein to fully fortified feeds may not only improve growth, but may also improve cognitive and motor outcomes.

9. Kuschel CA, Harding JE. Protein supplementation of human milk for promoting growth in preterm infants. Cochrane Database Syst Rev. 2000;2:CD000433.

10. - Young L, Morgan J, McCormick, FM, McGuire W. Nutrientenriched formula versus standard term formula for preterm infants following hospital discharge. Cochrane Database Syst Rev. 2012;3:CD004696. Many NICUs discharge preterm infants on nutrient-enriched ("postdischarge") formula feeds as standard of practice. However, a comprehensive of the data suggests that there is no neurocognitive benefit to this practice as currently formulated.

11. - Young L, Embleton ND, McCormick FM, McGuire W. Multinutrient fortification of human breast milk for preterm infants following hospital discharge. Cochrane Database Syst Rev 2013;2:CD004866. Many NICUs discharge preterm infants on nutrient-enriched breastmilk feeds as standard of practice. However, a comprehensive of the data suggests that although there is improvement in growth, there is no neurocognitive benefit to this practice as currently formulated.

12. ESPGHAN Committee on Nutrition, Aggett PJ, Agostoni C, et al. Feeding preterm infants after hospital discharge: a commentary by the ESPGHAN Committee on Nutrition. J Pediatr Gastroenterol Nutr. 2006;42:596-603.

13. Ambalavanan N, Nelson KG, Alexander G, et al. Prediction of neurologic morbidity in extremely low birth weight infants. J Perinatol. 2000;20(8 Pt 1):496-503.

14. Mitha A, Foix-L 'Helias L, Arnaud C, et al. Neonatal infection and 5-year neurodevelopmental outcome of very preterm infants. Pediatrics. 2013;132(2):e372-80.

15. - Schlapbach LJ, Aebischer M, Adams M, et al. Swiss Neonatal Network and Follow-up Group. Impact of sepsis on neurodevelopmental outcome in a Swiss national Cohort of extremely preterm infants. Pediatrics. 2011;128(2):e348-57. This study included a large number of participants and showed the independent effect of sepsis on neurodevelopmental outcomes.

16. Wu YW, Colford JM Jr. Chorioamnionitis as a risk factor for cerebral palsy: a meta-analysis. JAMA. 2000;284(11):1417-24.

17. Shah DK, Doyle LW, Anderson PJ, et al. Adverse neurodevelopment in preterm infants with postnatal sepsis or necrotizing enterocolitis is mediated by white matter abnormalities on magnetic resonance imaging at term. J Pediatr. 2008;153(2):170-5.

18. - Ramel SE, Demerath EW, Gray HL, et al. The relationship of poor linear growth velocity with neonatal illness and two-year neurodevelopment in preterm infants. Neonatology. 2012;102: 19-24. Growth of preterm infants is often defined as weight gain alone. This study looks specifically at the impact of linear growth during hospitalization and following discharge on later developmental outcomes.

19. Hansen-Pupp I, Hovel H, Lofqvist C, et al. Circulatory insulinlike growth factor-I and brain volumes in relation to neurodevelopmental outcomes in very preterm infants. Pediatr Res. 2013;74(5):564-9.

20. Lee KH, Calikoglu AS, Ye P, D'Ercole AJ. Insulin-like growth factor-I (IGF-I) ameliorates and IGF binding protein-1 (IGFBP-1) exacerbates the effects of undernutrition on brain growth during early postnatal life: studies in IGF-I and IGFBP-1 transgenic mice. Pediatr Res. 1999;45(3):331-6.

21. Miller BS, Kroupina MG, Mason P, et al. determinants of catchup growth in international adoptees from Eastern Europe. Int $\mathbf{J}$ Pediatr Endocrinol. 2010;2010:107252 Epub 2010 Dec 29.

22. Hulst JM, van Goudoever JB, Zimmermann LJ, et al. The effect of cumulative energy and protein deficiency on anthropometric parameters in a pediatric ICU population. Clin Nutr. 2004;23:1381-9.

23. Ehrenkranz RA, Dusick AM, Vohr BR, et al. Growth in the neonatal intensive care unit influences neurodevelopmental and growth outcomes of extremely low birth weight infants. Pediatrics. 2006;117:1253-61.

24. Franz AR, Pohlandt F, Bode H, et al. Intrauterine, early neonatal and post discharge growth and neurodevelopmental outcome at 5.4 years in extremely preterm infants after intensive nutritional support. Pediatrics. 2009;123:e101-9.

25. • Belfort MB, Rifas-Shiman SL, Sullivan T, et al. Infant growth before and after term: effects on neurodevelopment in preterm infants. Pediatrics. 2011;128:e899-906. This study not only included a large number of participants, it also looked at many measures of growth over several epochs of a preterm infants' life allowing for a very comprehensive look at preterm growth and outcomes.

26. Belfort MB, Gillman MW, Buka SL, Casey PH, McCormick MC. Preterm infant linear growth and adiposity gain: trade-offs for later weight status and intelligence quotient. J Pediatr. 2013;163(6):1564 9.e2.

27. Roggero $\mathrm{P}$, Gianní ML, Amato O, et al. Is term newborn body composition being achieved postnatally in preterm infants? Early Hum Dev. 2009;85:349-52.

28. Gianní ML, Roggero P, Taroni F, et al. Adiposity in small for gestational age preterm infants assessed at term equivalent age. Child Fetal Neonatal Ed. 2009;94:F368-72.

29. Pfister KM, Gray HL, Miller NC, et al. Exploratory study of the relationship of fat-free mass to speed of brain processing in preterm infants. Pediatr Res. 2013;74(5):576-83.

30. Ogden $\mathrm{C}$ and Carroll M. Prevalence of obesity among children and adolescents: United States trends 1963-1965 through 2007-2008. http://www.cdc.gov/nchs/data/hestat/obesity_child_ 07_08/obesity_child_07_08.htm.

31. Eriksson JG, Forsén T, Tuomilehto J, et al. Catch-up growth in childhood and death from coronary heart disease: longitudinal study. BMJ. 1999;318(7181):427-31.

32. Tinnion R, Gillone J, Cheetham T, Embleton N. Preterm birth and subsequent insulin sensitivity: a systematic review. Arch Dis Child. 2014;99:362-8.

33. de Jong F, Monuteaux MC, van Elburg RM, Gillman MW, Belfort MB. Systematic review and meta-analysis of preterm birth and later systolic blood pressure. Hypertension. 2012;59: 226-34. 
34. Kerkhof GF, Breukhoven PE, Leunissen RWJ, Willemsen RH, Hokken-Koelega ACS. Does preterm birth influence cardiovascular risk in early adulthood? J Pediatr. 2012;161:390-6.

35. - Parkinson JRC, Hyde MJ, Gale C, Santhakumaran, Modi N. Preterm birth and the metabolic syndrome in adult life: a systematic review and meta-analysis. Pediatrics. 2013;131:e1240-63. A comprehensive meta-analysis of influence of prematurity on many features of metabolic syndrome.

36. Ramel SE, Gray HL, Ode KL, et al. Body composition changes in preterm infants following hospital discharge: comparison with term infants. J Pediatr Gastroenterol Nutr. 2011;53:333-8.

37. Costa-Orvay JA, Figueras-Aloy J, Romera G, Closa-Monasterolo $\mathrm{R}$, Carbonell-Estrany X. The effects of varying protein and energy intakes on the growth and body composition of very low birth weight infants. Nutr J. 2011;10:140.

38. - Roggero P, Gianní ML, Amato O, et al. Growth and fat-free mass gain in preterm infants after discharge: a randomized controlled trial. Pediatrics. 2012;130:e1215-21. This study documents that fortified feeds in preterm infants after hospital discharge leads to improved growth without increasing body fatness.

39. Ludwig-Auser H, Sherar LB, Erlandson MC, et al. Influence of nutrition provision during the first two weeks of life in premature infants on adolescent body composition and blood pressure. Chin J Contemp Pediatr. 2013;15(3):161-70.

40. Singhal A, Fewtrell M, Cole TJ, Lucas A. Low nutrient intake and early growth for later insulin resistance in adolescents born preterm. Lancet. 2003;361(9363):1089-97.

41. Uthaya S, Thomas L, Hamilton G, et al. Altered adiposity after extreme preterm birth. Pediatr Res. 2005;57:211-5.

42. Johnson MJ, Wootton SA, Leaf AA, Jackson AA. Preterm birth and body composition at term equivalent age: a systematic review and meta-analysis. Pediatrics. 2012;130(3):e640-9.

43. Griffin IJ, Cooke RJ. Development of whole body adiposity in preterm infants. Early Hum Dev. 2012;88:S19-24.

44. Gianní ML, Mora S, Roggero P, et al. Regional fat distribution in children born preterm evaluated at school age. J Pediatr Gastroenterol Nutr. 2008;46:232-5.
45. Fewtrell MS, Lucas A, Cole TJ, Wells JC. Prematurity and reduced body fatness at $8-12$ y of age. Am J Clin Nutr. 2004;80(2):436-40.

46. Casey PH, Bradley RH, Whiteside-Mansell L, Barrett K, Gossett JM, Simpson PM. Evolution of obesity in a low birth weight cohort. J Perinatol. 2012;32:91-6.

47. Euser AM, Finken MJJ, Keijzer-Veen MG, et al. Associations between prenatal and infancy weight gain and BMI, fat mass, and fat distribution in young adulthood: a prospective cohort study in males and females born very preterm. Am J Clin Nutr. 2005;81:480-7.

48. Irving RJ, Belton NR, Elton RA, Walker BR. Adult cardiovascular risk factors in premature babies. Lancet. 2000;355:2135-6.

49. Keijzer-Veen MG, FInken MJ, Nauta J, et al. Is blood pressure increased 19 years after intrauterine growth restriction and preterm birth? A prospective follow-up study in the Netherlands. Pediatrics. 2005;116(3):725-31.

50. Hack M, Schluchter M, Carter L, Rahman M. Blood pressure among very low birth weight $(<1.5 \mathrm{~kg})$ young adults. Pediatr Res. 2005;58(4):677-84.

51. Belfort MB, Martin CR, Smith VC, Gillman MW, McCormick MC. Infant weight gain and school-age blood pressure and cognition in former preterm children. Pediatr. 2010;125:e1419.

52. Fewtrell MS, Doherty C, Cole TJ, Stafford M, Hales CN, Lucas A. Effects of size at birth, gestational age and early growth in preterm infants on glucose and insulin concentrations at 9-12 years. Diabetol. 2000;43:714-7.

53. Finken MJ, Inderson A, Van Montfoort N, et al. Lipid profile and carotid intima-media thickness in a prospective cohort of very preterm subjects at age 19 years: effects of early growth and current body composition. Pediatr Res. 2006;59:604-9.

54. Hovi P, Andersson S, Eriksson JG, et al. Glucose regulation in young adults with very low birth weight. $\mathrm{N}$ Eng J Med. 2007;356:2053-63. 